\title{
Wireless Echo Friendly Communication System for Future Broad Band Applications
}

\author{
K.Murali $^{\#}$, K.Prasuna \\ \# ECE Department, Vijaya Institute of Technology for Women, Vijayawada (A.P.), India \\ E-mail: kalipindimurali@gmail.com
}

\begin{abstract}
Our aim is to build an eco-friendly system for the assortment of information from the environment. The system must be power-independent with the usage of renewable power bases. As a holdup different resources in power source in the event of critical conditions, other ecological technologies can be used. Our proposed system needs to be power efficient and should be able to accomplish itself without any exterior observation.
\end{abstract}

Keywords - eco-friendly, renewable power, ecological technologies, power efficient.

\section{INTRODUCTION}

The fast emergence of contemporary data and wireless technologies offer a chance to strongly analyse the situation around us. The situation can be analysed various environments [1]. In this Paper, the proposed system is echo friendly. This can be achieved by projecting out typical power source which includes hazardous to the situation. Instead of power source, other sources may be combined in such a way that the critical conditions would not affect power failure. This leads to the necessity of storage of power, making system operation efficient.

\section{OVER VIEW OF THE PAPER}

This paper provides the best trade off between rate constraints and power constraints. In section I we discuss the introduction with certain aspects. In section 3 possible of echo friendly wireless communication system basic scenario. In section 4 we provide the basic over view about the different wireless generations. In section 5 we discuss the applications and advantages of our proposed system. In section 6 we discuss the conclusions.

\section{WIRELESS ECHO FRIENDLY SYSTEM}

4G encompasses in jamming signals so that data can be attained unlawfully in a easy way.4G Technology is cost expensive and complicated to implement. It devours a lot of battery and becomes hot when it is used for a extensive time. 4G LTE network necessities multifaceted hardware.it uses many antennas and transmitters. It is still inadequate to definite areas and cities, which takes a lot of time. $4 \mathrm{G}$ is ideal for data rates, but not inevitably the best for Voice services [2]. Wireless Echo Friendly Communication System overcomes the above disadvantages. The main objective of echo friendly Communication system is to provide

1. a higher speed of internet at a lesser cost

2. to reduce battery drainage, lower latency

3 . to increase the security and

4. Connectivity for a large community.

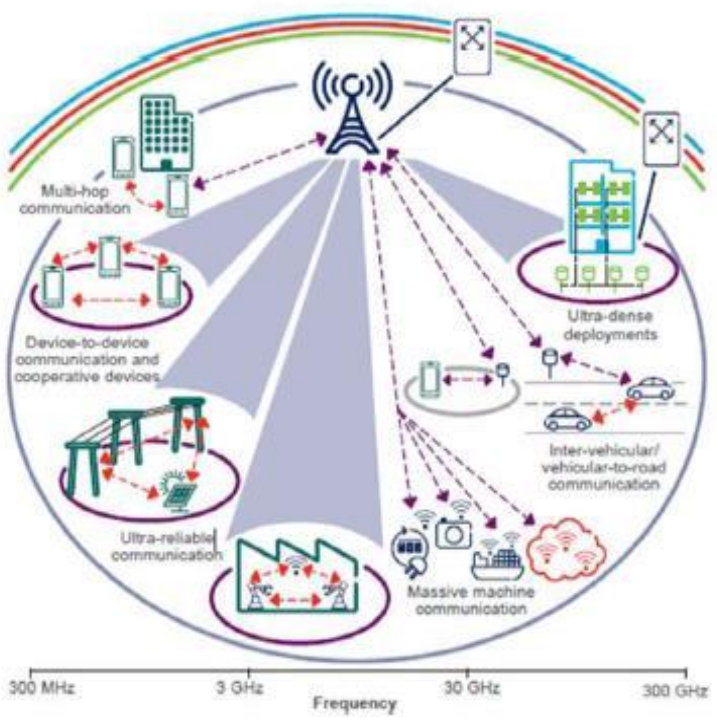

Fig.1 Scenario of Echo Friendly Environment 


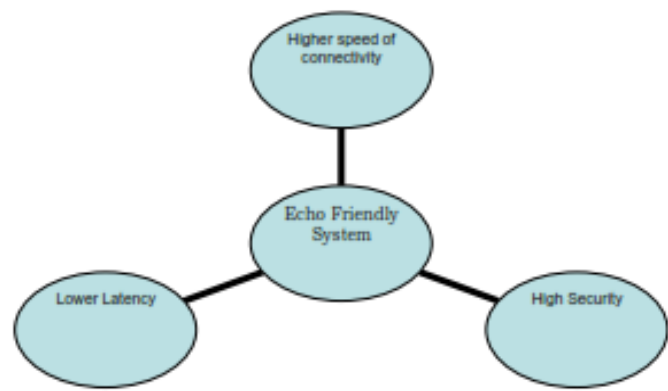

Fig.2 Features of Echo Friendly Environment

\section{CURRENT WIRELESS TECHNOLOGIES}

As the different Technologies of wireless have evolved, each one has conveyedits own enhancements. The same will be true of Wireless Echo Friendly Communication Technology.

1. First generation, 1G: This generation is analogue and offers low spectrum efficiency.

2. Second generation, 2G: This generation provides digital components, which enhances rate structure.

3. Third generation, 3G: This generation offers high speed data and consistent to statistics

4. Fourth generation, 4G: This generation is an IP based skill generation offers high data rate.

Any new 5th generation, 5G cellular technology needs to offer important gains in excess of earlier systems to give an enough industry box for portable operator to invest in any new system. Wireless Echo Friendly Communication Technology provides for improved connectivity and Coverage. This Technology has to address on lack of coverage, dropped calls and low performance at cell edges.

\section{ApPlication Possibilities AND AdVANTAGEs OF THE SYSTEM}

It is possible to formulate a number of areas in which our concept of wireless eco-friendly Communication System can be totally realized:
Monitoring of Environmental Quality - where relevant data (e.g. pollution levels, the presence of hazardous elements, etc.) is mostly collected at the monitoring stations. Monitoring of meteorological parameters

- will help to produce quality information on the meteorological situation in the area. It can help to identify the conditions for the protection of forests and countries, but also in the evaluation of conditions for overpopulation of pests in forests. [5] Monitoring of wildlife migration The issue of wildlife relocation monitoring is especially important for animal species protection. Based on the knowledge of the territory it is possible to create new protected areas or increase/decrease the existing level of protection. Forest fires, earthquakes, landslides, volcanoes, floods Detection, Alert and early warning mechanism, Health Internet of Things.

\section{CONCLUSIONS}

Our conclusion is that data rate should be increased with minimal power consumption. so, Solar cell panel size should be reduced with lower latency and security should be increased. The Connectivity for a large community should be maintained.

\section{REFERENCES}

[1] M. Bloch and J. Barros, Physical-Layer Security: From Information Theory to Security Engineering. Cambridge University Press, 2011.

[2] X. Zhou, L. Song, and Z. Zhang, Physical Layer Security in Wireless Communications. CRC Press, 2013.

[3] A. Mukherjee, S. Fakoorian, J. Huang, and A. L. Swindlehurst, "Principles of physical layer security in multiuser wireless networks: A survey," IEEE Commun. Surveys Tuts., vol. 16, no. 3 , pp. 1550-1573, Aug. 2014.

[4] L. Dong, Z. Han, A. P. Petropulu, and H. V. Poor, "Amplify-andforward based cooperation for secure wireless communications," in Proc. IEEE ICASSP, Apr. 2009, pp. 2613-2616.

[5] V. Aggarwal, L. Sankar, A. Calderbank, and H. Poor, "Secrecy capacity of a class of orthogonal relay eavesdropper channels," EURASIP J. Wireless Commun. Netw., vol. 2009, Jul.2009 